MITSUBISHI ELECTRIC RESEARCH LABORATORIES

http://www.merl.com

\title{
State-of-Charge Estimation of Lithium-Ion Batteries via a Coupled Thermal-Electrochemical Model
}

\author{
Tang, S.; Wang, Y.; Sahinoglu, Z.; Wada, T.; Hara, S.; Krstic, M.
}

TR2015-065 July 2015

\begin{abstract}
Accurate online state-of-charge ( $\mathrm{SoC})$ estimation is a basic need and also a fundamental challenge for battery applications. In order to achieve accurate SoC estimation for the lithiumion batteries, we employ a coupled thermal-electrochemical model. This coupled system of an ordinary differential equation (ODE) and a partial differential equation (PDE) is simpler than the Doyle-Fuller-Newman (DFN) model, and is more accurate than the single particle model (SPM) alone. Thus, it could serve as a better fit of model for a full state observer design and accurate SoC estimation. PDE backstepping approach is utilized to develop a Luenberger observer for the electrode concentration, and estimation effectiveness of the proposed method is verified by simulation results.
\end{abstract}

2015 American Control Conference (ACC)

\footnotetext{
This work may not be copied or reproduced in whole or in part for any commercial purpose. Permission to copy in whole or in part without payment of fee is granted for nonprofit educational and research purposes provided that all such whole or partial copies include the following: a notice that such copying is by permission of Mitsubishi Electric Research Laboratories, Inc.; an acknowledgment of the authors and individual contributions to the work; and all applicable portions of the copyright notice. Copying, reproduction, or republishing for any other purpose shall require a license with payment of fee to Mitsubishi Electric Research Laboratories, Inc. All rights reserved.
} 



\title{
State-of-Charge Estimation for Lithium-Ion Batteries via a Coupled Thermal-Electrochemical Model
}

\author{
Shuxia Tang, Yebin Wang, Zafer Sahinoglu, Toshihiro Wada, Satoshi Hara and Miroslav Krstic
}

\begin{abstract}
Accurate online state-of-charge (SoC) estimation is a basic need and also a fundamental challenge for battery applications. In order to achieve accurate SoC estimation for the lithium-ion batteries, we employ a coupled thermalelectrochemical model. This coupled system of an ordinary differential equation (ODE) and a partial differential equation (PDE) is simpler than the Doyle-Fuller-Newman (DFN) model, and is more accurate than the single particle model (SPM) alone. Thus, it could serve as a better fit of model for a full state observer design and accurate SoC estimation. PDE backstepping approach is utilized to develop a Luenberger observer for the electrode concentration, and estimation effectiveness of the proposed method is verified by simulation results.
\end{abstract}

\section{INTRODUCTION}

Rechargeable batteries are receiving more and more extensive attention as an energy source in portable electronics, electric vehicles (EV), hybrid electric vehicles (HEV) and smart grid storage. The lithium-ion battery, a type of rechargeable battery, is now a common choice in these application areas because of its high energy storage density, lack of memory effect, low self discharge, and high cycle life. Accurate on-line estimation of the lithium-ion battery SoC, i.e., the percentage ratio of the instantaneous battery capacity to its nominal/maximum capacity, is expected in order to predict power and energy available in the battery during operation. It also serves as an important factor for regulating charging/discharging in real time.

Electrochemical models, such as the DFN model [4] and SPM, can provide a fundamental understanding of internal processes in the battery and can accurately predict state variables. DFN model is formulated by modeling the process of ion intercalation, that is, of ions moving in and out of interstitial site in the lattices in each electrode. However, it is too complex for the researchers to design observer and conduct observability analysis [5]. SPM [6], [7] is an approximation/reduction of the full DFN model. It is formulated by idealizing the solid phase of each electrode as a single spherical particle and assuming the electrolyte concentration to be uniform in both space and time. Thus,

S. Tang and M. Krstic are with Department of Mechanical and Aerospace Engineering, University of California, San Diego, La Jolla, CA 92093, USA. sht015@ucsd.edu; krstic@ucsd.edu. This work was carried out during S. Tang's visit to Mitsubishi Electric Research Laboratories, 201 Broadway, Cambridge, MA 02139, USA

Y. Wang and Z. Sahinoglu are with Mitsubishi Electric Research Laboratories, 201 Broadway, Cambridge, MA 02139, USA. yebingwang@ieee.org; zafer@merl.com.

T. Wada and S. Hara are with the Advanced Technology R\&D Center, Mitsubishi Electric Corporation, 8-1-1, Tsukaguchi honmachi, Amagasaki City, 661-8661, Japan. Wada.Toshihi-ro@bx.MitsubishiE-lectric.co.jp; Hara.Satoshi@cb.MitsubishiElectric.co.jp. it is simple enough for the engineers to design an observer and conduct rigorous observability analysis, but it could only produce accurate $\mathrm{SoC}$ estimation for the low current case, i.e. low C-rate.

The SPM [8] assumes a constant temperature. However, electrochemical and chemical processes inside the battery cell during charging/discharging have their impacts on the thermal behavior [9], [10]. More importantly, temperature could also influence the battery performance since the model parameters are physically dependent on the battery temperature, for which we refer the readers to [11], [12], [13]. In this paper, we take the thermal behavior into account and consider a coupled thermal and electrochemical model, which consists of an ODE for the internal average temperature dynamics and a diffusion-advection PDE for dynamics of the lithium-ion concentration in each electrode.

The method of PDE backstepping can be used for stabilizing unstable PDE systems, see [14], in which backstepping boundary controllers and observers are designed for some unstable parabolic, hyperbolic PDEs, etc. It can also be applied for stabilizing some coupled PDE-ODE systems [15], [16]. This paper employs the PDE backstepping method to design a full-state observer through boundary state measurement for the coupled PDE-ODE system.

This paper is organized as follows. In Section II, a temperature-compensated model (SPM-T) is presented and the SoC estimation problem is formulated. In Section III, a backstepping state observer is designed and the observer error system is proved to be exponentially stable with an arbitrarily designated decay rate. Numerical simulations are performed in Section IV, and the results verify effectiveness of the estimation method. Moreover, a conclusion and some possible future works are given in Section V.

\section{PROBLEM FORMULATION}

\section{A. An SPM-T Model}

1) System equations: Consider the following coupled system of an SPM model [8]

$$
\begin{aligned}
& \frac{\partial c_{s}^{ \pm}}{\partial t}\left(r_{s}, t\right)=\frac{1}{r_{s}^{2}} \frac{\partial}{\partial r_{s}}\left(D_{s}^{ \pm}(T(t)) r_{s}^{2} \frac{\partial c_{s}^{ \pm}}{\partial r_{s}}\left(r_{s}, t\right)\right), \\
& r_{s} \in\left(0, R_{s}^{ \pm}\right), t>0 \\
& \frac{\partial c_{s}^{ \pm}}{\partial r_{s}}(0, t)=0, t>0 \\
& \frac{\partial c_{s}^{ \pm}}{\partial r_{s}}\left(R_{s}^{ \pm}, t\right)=-\frac{1}{D_{s}^{ \pm}(T(t))} J^{ \pm}(t), t>0 \\
& c_{s}^{ \pm}\left(r_{s}, 0\right)=c_{s 0}^{ \pm}\left(r_{s}\right), r_{s} \in\left(0, R_{s}^{ \pm}\right)
\end{aligned}
$$


with an internal average temperature model [10]

$$
\begin{aligned}
& \rho^{\mathrm{avg}} C_{P} \frac{d T}{d t}(t)=h_{\mathrm{cell}}\left(T_{\mathrm{amb}}(t)-T(t)\right)+I(t) V(t) \\
& \quad-a_{s}^{-} F J^{-}(t) \Delta U^{-}(t) L^{-}-a_{s}^{+} F J^{+}(t) \Delta U^{+}(t) L^{+}, t>0 \\
& T(0)=T_{a m b}(0) .
\end{aligned}
$$

To better understand SPM, the readers could refer to [4] for more details, which are omitted here due to space limitation.

2) System \& problem demonstration: Standard international units are used to quantify parameters, functions and states through this paper. The spatial variable $r_{s} \in\left[0, R_{s}^{ \pm}\right]$, the time variable $t \in[0, \infty)$. The states of the PDE model $(1)-(6)$ are $c_{s}^{ \pm}\left(r_{s}, t\right) \in \mathbb{R}$, which denote the solid phase lithium-ion concentration ( + for positive electrode; - for negative electrode), and the ODE state is $T(t)$, which denotes the internal average temperature. The input to the entire coupled system is the applied current density $I(t)$, and the model output is the closed-loop voltage $V(t)$, which is the potential difference measured across the current collectors at the external boundaries of the electrodes. The objective is to estimate the bulk SoC on-line, which is defined by:

$$
\operatorname{SoC}^{ \pm}(t)=\frac{3}{\left(R_{s}^{ \pm}\right)^{3}} \int_{0}^{R_{s}^{ \pm}} r^{2} \frac{c_{s}^{ \pm}(r, t)}{c_{s, \max }^{ \pm}} d r
$$

Diffusion coefficient functions for the PDE (1) - (4) are considered to be of an Arrhenius-like dependency on temperature [5]:

$$
D_{s}^{ \pm}(T(t))=D_{s}^{ \pm}\left(T_{0}\right) e^{A D_{s}^{ \pm} \frac{T(t)-T_{0}}{T(t) T_{0}}}
$$

where $A_{D_{s}^{ \pm}}$are constant model parameters. $J^{ \pm}$are the pore wall fluxes at the surface of the particles, and are related to the current $I(t)$ :

$$
J^{+}(t)=-\frac{I(t)}{a_{s}^{+} A F L^{+}}, J^{-}(t)=\frac{I(t)}{a_{s}^{-} A F L^{-}},
$$

where $a_{s}^{ \pm}$are specific interfacial surface areas, $A$ is the cell cross sectional area, $F$ is the Faraday's constant, and $L^{ \pm}$ define the electrodes' thickness.

In the ODE (5) - (6), $\rho^{\text {avg }}$ is the lumped density, $c_{P}$ is the heat capacity, and $h_{\text {cell }}$ is the heat transfer coefficient. $T_{\mathrm{amb}}(t)$ is the ambient temperature, and $\Delta U^{ \pm}(t)$ are defined as

$$
\Delta U^{ \pm}(t)=U^{ \pm}\left(\bar{c}_{s}^{ \pm}(t)\right)-T(t) \frac{\partial U^{ \pm}\left(\bar{c}_{s}^{ \pm}(t)\right)}{\partial T}
$$

where $U^{ \pm}$denote the reaction potentials and

$$
\bar{c}_{s}^{ \pm}(t)=\frac{3}{\left(R_{p}^{ \pm}\right)^{3}} \int_{0}^{R_{p}^{ \pm}} r_{s}^{2} c_{s}^{ \pm}\left(r_{s}, t\right) d r_{s}
$$

denote the volume averaged solid phase concentration. Thus, the temperature model (5)-(6) is rewritten as follows

$$
\begin{aligned}
& \rho^{\mathrm{avg}} C_{P} \frac{d T}{d t}(t)=h_{\text {cell }}\left(T_{\mathrm{amb}}(t)-T(t)\right)+I(t) V(t) \\
&+ \frac{1}{A} I(t)\left[U^{+}\left(\bar{c}_{s}^{+}(t)\right)-U^{-}\left(\bar{c}_{s}^{-}(t)\right)\right. \\
&-\left.T(t) \frac{\partial\left(U^{+}\left(\bar{c}_{s}^{+}(t)\right)-U^{-}\left(\bar{c}_{s}^{-}(t)\right)\right)}{\partial T}\right], t>0 \\
& T(0)=T_{a m b}(0)
\end{aligned}
$$

3) System output function formula derivation: Next, we are to derive the output equation for $V(t)$. Firstly, the overpotential equations for the ion intercalation reaction are

$$
\begin{aligned}
\eta^{ \pm}(t)= & \phi_{s}^{ \pm}\left(0^{ \pm}, t\right)-\phi_{e}\left(0^{ \pm}, t\right) \\
& -U^{ \pm}\left(c_{s s}^{ \pm}(t)\right)-F R_{f}^{ \pm}(T(t)) J^{ \pm}(t),
\end{aligned}
$$

where $\phi_{s}^{ \pm}\left(0^{ \pm}, t\right)$ and $\phi_{e}\left(0^{ \pm}, t\right)$ denote the electric potentials at current collectors in solid electrodes and electrolyte respectively; $c_{s s}^{ \pm}(t) \triangleq c_{s}^{ \pm}\left(R_{s}^{ \pm}, t\right)$; the film resistance of the solidelectrolyte interphase (SEI) is

$$
R_{f}^{ \pm}(T(t))=R_{f}^{ \pm}\left(T_{0}\right) e^{A_{R_{f}}^{ \pm} \frac{T(t)-T_{0}}{T(t) T_{0}}}
$$

with $A_{R_{f}}^{ \pm}$being constants. Thus, the voltage is derived as

$$
\begin{aligned}
V(t)= & \phi_{s}^{+}\left(0^{+}, t\right)-\phi_{s}^{-}\left(0^{-}, t\right) \\
= & \eta^{+}(t)-\eta^{-}(t)+\phi_{e}\left(0^{+}, t\right)-\phi_{e}\left(0^{-}, t\right) \\
& -\left(\frac{R_{f}^{+}(T(t))}{a^{+} A L^{+}}+\frac{R_{f}^{-}(T(t))}{a^{-} A L^{-}}\right) I(t) \\
& +U^{+}\left(c_{s s}^{+}(t)\right)-U^{-}\left(c_{s s}^{-}(t)\right) .
\end{aligned}
$$

Secondly, the Butler-Volmer dynamics is

$$
J^{ \pm}(t)=\frac{i_{0}^{ \pm}(t)}{F}\left(e^{\frac{\alpha_{a} F}{R T(t)} \eta^{ \pm}(t)}-e^{-\frac{\alpha_{c} F}{R T(t)} \eta^{ \pm}(t)}\right)
$$

where we assume $\alpha_{a}=\alpha_{c}=\alpha$, and the exchange current densities are given by

$$
i_{0}^{ \pm}(t)=r_{e f f}^{ \pm}(T(t))\left(c_{e}\left(c_{s}^{ \pm, \max }-c_{s}^{ \pm}\left(R_{s}^{ \pm}, t\right)\right) c_{s}^{ \pm}\left(R_{s}^{ \pm}, t\right)\right)^{\alpha}
$$

with

$$
r_{e f f}^{ \pm}(T(t))=r_{e f f}^{ \pm}\left(T_{0}\right) e^{A_{r e f f} \frac{T(t)-T_{0}}{T(t) T_{0}}}
$$

Here $c_{e}$ denotes the constant electrolyte concentration, $c_{s}^{ \pm, \max }$ are the maximum possible concentration of lithium in the solid particles of electrodes, $A_{r_{e} f f}$ is constant. Then,

$$
\eta^{ \pm}(t)=\frac{R T(t)}{\alpha F} \sinh ^{-1}\left(\frac{F}{2 i_{0}^{ \pm}(t)} J^{ \pm}(t)\right) .
$$

From the assumption that the electrolyte Li concentration is constant in space and time, it holds that

$$
\phi_{e}\left(0^{+}, t\right)=\phi_{e}\left(0^{-}, t\right) \text {. }
$$


Thus, from $(10),(11),(12)$, the following system output function is derived:

$$
\begin{aligned}
V(t)= & -\frac{R T(t)}{\alpha F}\left[\sinh ^{-1}\left(\frac{1}{2 i_{0}^{+}(t)} \frac{I(t)}{a^{+} A L^{+}}\right)\right. \\
& \left.+\sinh ^{-1}\left(\frac{1}{2 i_{0}^{-}(t)} \frac{I(t)}{a^{-} A L^{-}}\right)\right] \\
& -\left(\frac{R_{f}^{+}(T(t))}{a^{+} A L^{+}}+\frac{R_{f}^{-}(T(t))}{a^{-} A L^{-}}\right) I(t) \\
& +U^{+}\left(c_{s s}^{+}(t)\right)-U^{-}\left(c_{s s}^{-}(t)\right) \\
\triangleq & h_{1}\left(T(t), c_{s s}^{+}(t), c_{s s}^{-}(t), I(t)\right) .
\end{aligned}
$$

\section{BACKSTEPPing StATE OBSERVER}

Since the output function $V(t)$, as in (13), is a nonlinear function of the states, we would like to seek other measurement variables so that observability analysis could be proceeded and a linear PDE observer could be designed. Boundary concentration is an excellent choice, and one idea is to get the boundary concentration information from the measured output $V(t)$. Great difficulty lies in that there are too many unknown elements in (13). In what follows, we will derive a simplified formula for $V(t)$, in which $V(t)$ only relies on $c_{s S}^{-}(t)$ and input $I(t)$, then we only need to consider the anode PDE for SoC estimation.

\section{A. Deriving anode boundary concentration from measured} output voltage

1) Write $V(t)$ as a function of $c_{s s}^{ \pm}(t)$ and $\bar{c}_{s}^{ \pm}(t)$ : By substituting (13) into (8), we have

$$
\begin{aligned}
\rho^{\mathrm{avg}} c_{P} \frac{d T}{d t}(t)= & h_{\mathrm{cell}}\left(T_{\mathrm{amb}}(t)-T(t)\right) \\
& -I(t) \frac{R T(t)}{\alpha F}\left[\sinh ^{-1}\left(\frac{1}{2 i_{0}^{+}(t)} \frac{I(t)}{a^{+} A L^{+}}\right)\right. \\
& \left.+\sinh ^{-1}\left(\frac{1}{2 i_{0}^{-}(t)} \frac{I(t)}{a^{-} A L^{-}}\right)\right] \\
& -\left(\frac{R_{f}^{+}(T(t))}{a^{+} A L^{+}}+\frac{R_{f}^{-}(T(t))}{a^{-} A L^{-}}\right) I(t)^{2} \\
& +I(t)\left(U^{+}\left(c_{s s}^{+}(t)\right)-U^{-}\left(c_{s s}^{-}(t)\right)\right) \\
& +\frac{1}{A} I(t)\left[U^{+}\left(\bar{c}_{s}^{+}(t)\right)-U^{-}\left(\bar{c}_{s}^{-}(t)\right)\right. \\
& \left.-T(t) \frac{\left.\partial\left(U^{+}\left(\bar{c}_{s}^{+}(t)\right)-U^{-}\left(\bar{c}_{s}^{-}(t)\right)\right)\right]}{\partial T}\right]
\end{aligned}
$$

$R_{f}^{ \pm}, r_{e f f}^{ \pm}$are functions of $T(t)$, of which the exact forms will be used in simulation and experiments. However, we assume them to be constant for analysis convenience here. If also assuming $\frac{\partial\left(U^{+}\left(\bar{c}_{s}^{+}(t)\right)-U^{-}\left(\bar{c}_{s}^{-}(t)\right)\right)}{\partial T}$ to be a state-invariant, possibly time-varying, function, then analytical solution is available. Write

$$
\rho^{\mathrm{avg}} c_{P} \frac{d T}{d t}(t)=\chi(t) T(t)+\omega(t)
$$

where

$$
\begin{aligned}
\chi(t)= & -h_{\text {cell }}-\frac{R}{\alpha F} I(t)\left[\sinh ^{-1}\left(\frac{1}{2 i_{0}^{+}(t)} \frac{I(t)}{a^{+} A L^{+}}\right)\right. \\
& \left.+\sinh ^{-1}\left(\frac{1}{2 i_{0}^{-}(t)} \frac{I(t)}{a^{-} A L^{-}}\right)\right] \\
& -\frac{1}{A} I(t) \frac{\partial\left(U^{+}\left(\bar{c}_{s}^{+}(t)\right)-U^{+}\left(\bar{c}_{s}^{+}(t)\right)\right)}{\partial T} \\
\omega(t)= & h_{\mathrm{cell}} T_{\mathrm{amb}}(t)-\left(\frac{R_{f}^{+}}{a^{+} A L^{+}}+\frac{R_{f}^{-}}{a^{-} A L^{-}}\right) I(t)^{2} \\
& +I(t)\left(U^{+}\left(c_{s s}^{+}(t)\right)-U^{-}\left(c_{s s}^{-}(t)\right)\right) \\
& +\frac{1}{A} I(t)\left(U^{+}\left(\bar{c}_{s}^{+}(t)\right)-U^{-}\left(\bar{c}_{s}^{-}(t)\right)\right)
\end{aligned}
$$

Then

$$
\begin{aligned}
T(t)= & T_{0} e^{\frac{1}{\rho^{\operatorname{avg} c_{P}}} \int_{0}^{t} \chi(\tau) d \tau} \\
& +\frac{1}{\rho^{\operatorname{avg}_{C_{P}}}} \int_{0}^{t} e^{\frac{1}{\rho^{\operatorname{avg} c_{P}}} \int_{0}^{t-\tau} \chi(\sigma) d \sigma} \omega(\tau) d \tau
\end{aligned}
$$

Substituting (14) into (13) yields the following simplified output function

$$
\begin{aligned}
V(t)= & -\frac{R}{\alpha F}\left[\sinh ^{-1}\left(\frac{1}{2 i_{0}^{+}(t)} \frac{I(t)}{a^{+} A L^{+}}\right)\right. \\
& \left.+\sinh ^{-1}\left(\frac{1}{2 i_{0}^{-}(t)} \frac{I(t)}{a^{-} A L^{-}}\right)\right] \times\left(T_{0} e^{\frac{1}{\rho^{\operatorname{avg} c_{P}}} \int_{0}^{t} \chi(\tau) d \tau}\right. \\
& \left.+\frac{1}{\rho^{\text {avg }} c_{P}} \int_{0}^{t} e^{\frac{1}{\rho^{a v g} c_{P}} \int_{0}^{t-\tau} \chi(\sigma) d \sigma} \omega(\tau) d \tau\right) \\
& -\left(\frac{R_{f}^{+}}{a^{+} A L^{+}}+\frac{R_{f}^{-}}{a^{-} A L^{-}}\right) I(t)+U^{+}\left(c_{s s}^{+}(t)\right)-U^{-}\left(c_{s s}^{-}(t)\right) \\
= & h_{2}\left(c_{s s}^{ \pm}(t), \bar{c}_{s}^{ \pm}(t), I(t)\right)
\end{aligned}
$$

2) Write $V(t)$ as a function of $c_{s s}^{-}(t)$ : In order to further simplify the output function, we are to establish relations between $c_{s s}^{+}(t), \bar{c}_{s}^{+}(t), \bar{c}_{s}^{-}(t)$ and $c_{s s}^{-}(t)$ from an approximate solution. Consider the following approximate polynomial solution profiles of the electrode diffusion dynamics [17],

$$
\bar{c}_{s}^{ \pm}(t)=c_{s s}^{ \pm}(t)-\frac{8 R_{s}^{ \pm}}{35} \bar{q}_{s}^{ \pm}(t)+\frac{R_{s}^{ \pm}}{35 D_{s}^{ \pm}(T(t))} J^{ \pm}(t),
$$

where the volume averaged fluxes $\bar{q}_{s}^{ \pm}(t)$ satisfy

$$
\frac{d}{d t} \bar{q}_{s}^{ \pm}(t)=-\frac{30 D_{s}^{ \pm}(T(t))}{\left(R_{s}^{ \pm}\right)^{2}} \bar{q}_{s}^{ \pm}(t)-\frac{45}{2\left(R_{s}^{ \pm}\right)^{2}} J^{ \pm}(t),
$$

and $T(t)$ is from the following simplified model

$$
\rho^{\mathrm{avg}} c_{P} \frac{d T}{d t}(t)=h_{\text {cell }}\left(T_{\mathrm{amb}}(t)-T(t)\right)+I(t) V(t) .
$$

Since the total number of lithium ions is conserved in this model, that is,

$$
n_{L i}=\varepsilon^{+} L^{+} A \bar{c}_{s}^{+}(t)+\varepsilon^{-} L^{-} A \bar{c}_{s}^{-}(t),
$$

then from (16), it could be obtained that

$$
\begin{aligned}
& \bar{c}_{s}^{+}(t)=\alpha \bar{c}_{s}^{-}(t)+\beta \\
& =\alpha\left(c_{s s}^{-}(t)-\frac{8 R_{s}^{-}}{35} \bar{q}_{s}^{-}(t)+\frac{R_{s}^{-}}{35 D_{s}^{-}(T(t))} J^{-}(t)\right)+\beta,
\end{aligned}
$$


and

$$
\begin{aligned}
c_{s S}^{+}(t)= & \bar{c}_{s}^{+}(t)+\frac{8 R_{s}^{+}}{35} \bar{q}_{s}^{+}(t)-\frac{R_{s}^{+}}{35 D_{s}^{+}(T(t))} J^{+}(t) \\
= & \alpha\left(c_{s s}^{-}(t)-\frac{8 R_{s}^{-}}{35} \bar{q}_{s}^{-}(t)+\frac{R_{s}^{-}}{35 D_{s}^{-}(T(t))} J^{-}(t)\right)+\beta \\
& +\frac{8 R_{s}^{+}}{35} \bar{q}_{s}^{+}(t)-\frac{R_{s}^{+}}{35 D_{s}^{+}(T(t))} J^{+}(t)
\end{aligned}
$$

where

$$
\alpha=-\frac{\varepsilon^{-} L^{-}}{\varepsilon^{+} L^{+}}, \beta=\frac{n_{L i}}{\varepsilon^{+} L^{+} A} .
$$

The value of $n_{L i}$ could be calculated from the model initial data.

Therefore, from $(15),(16),(18),(19)$, we could obtain a further simplified output function

$$
V(t)=h_{3}\left(c_{s s}^{-}(t), I(t)\right) \text {. }
$$

3) Invert the simplified nonlinear output function: As long as the function (20) is a one-to-one function w.r.t. $c_{s s}^{-}(t)$, uniformly in $I(t)$, we could invert it to derive anode boundary concentration as

$$
c_{s s}^{-}(t)=h_{0}(V(t), I(t)) .
$$

\section{B. Backstepping boundary observer}

1) Normalization and state transformation: We perform normalization and state transformation to simplify the observer structure. Let $r=\frac{r_{s}}{R_{s}^{-}}$for normalization and proceed the state transformation $c(r, t)=r_{s} c_{s}^{-}\left(r_{s}, t\right)$, then the PDE system part (1) - (4) of the model is transformed into

$$
\begin{aligned}
& \frac{\partial c}{\partial t}(r, t)=\frac{D_{s}^{-}(T(t))}{\left(R_{s}^{-}\right)^{2}} \frac{\partial^{2} c}{\partial r^{2}}(r, t), r \in(0,1), t>0 \\
& c(0, t)=0, t>0 \\
& \frac{\partial c}{\partial r}(1, t)-c(1, t)=-\frac{R_{s}^{-}}{D_{s}^{-}(T(t))} \frac{I(t)}{a^{-} A F L^{-}}, t>0 \\
& c(r, 0)=c_{0}(r)=R_{s}^{-} r c_{s}^{-}\left(R_{s}^{-} r, 0\right), r \in(0,1]
\end{aligned}
$$

2) Observer design: With inversion of the output function in Section III-A, the anode boundary concentration is available for the observer design. In the mean time, $T(t)$ is calculated from the simplified equation (17), and thus, $D_{s}^{-}(T(t))$ could be treated as known. Hence, a Luenbergertype observer for the normalized and transformed PDE system (21) - (24) with boundary state error injection could be designed:

$$
\begin{aligned}
& \frac{\partial \hat{c}}{\partial t}(r, t)=\frac{D_{s}^{-}(T(t))}{\left(R_{s}^{-}\right)^{2}} \frac{\partial^{2} \hat{c}}{\partial r^{2}}(r, t) \\
& +p_{1}(r, t)(c(1, t)-\hat{c}(1, t)), r \in(0,1), t>0 \\
& \hat{c}(0, t)=0, t>0 \\
& \frac{\partial \hat{c}}{\partial r}(1, t)-\hat{c}(1, t)=-\frac{R_{s}^{-}}{D_{s}^{-}(T(t))} \frac{I(t)}{a^{-} A F L^{-}} \\
& +p_{10}(t)(c(1, t)-\hat{c}(1, t)), t>0 \\
& \hat{c}(r, 0)=\hat{c}_{0}(r), r \in(0,1],
\end{aligned}
$$

where the functions $p_{1}(r, t)$ and $p_{10}(t)$ are to be determined to guarantee the stability of the following observer error $(\tilde{c}(r, t)=c(r, t)-\hat{c}(r, t))$ system:

$$
\begin{aligned}
& \frac{\partial \tilde{c}}{\partial t}(r, t)=\frac{D_{s}^{-}(T(t))}{\left(R_{s}^{-}\right)^{2}} \frac{\partial^{2} \tilde{c}}{\partial r^{2}}(r, t) \\
& -p_{1}(r) \tilde{c}(1, t), r \in(0,1), t>0 \\
& \tilde{c}(0, t)=0, t>0 \\
& \frac{\partial \tilde{c}}{\partial r}(1, t)-\tilde{c}(1, t)=-p_{10} \tilde{c}(1, t), t>0 .
\end{aligned}
$$

In order to find the output injection gains, the PDE backstepping method [14] is employed. We would like to find an invertible transformation

$$
\tilde{c}(r, t)=\tilde{w}(r, t)-\int_{r}^{1} p(r, s, t) \tilde{w}(s, t) d s
$$

so that $\tilde{w}$ satisfies the following exponentially stable system

$$
\begin{aligned}
& \frac{\partial \tilde{w}}{\partial t}(r, t)=\frac{D_{s}^{-}(T(t))}{\left(R_{s}^{-}\right)^{2}} \frac{\partial^{2} \tilde{w}}{\partial r^{2}}(r, t)+\lambda \tilde{w}(r, t), \\
& \quad r \in(0,1), t>0 \\
& \tilde{w}(0, t)=0, t>0 \\
& \frac{\partial \tilde{w}}{\partial r}(1, t)=-\frac{1}{2} \tilde{w}(1, t), t>0,
\end{aligned}
$$

where $\lambda<\frac{1}{4\left(R_{s}^{-}\right)^{2}} \min _{t \geq 0} D_{s}^{-}(T(t))$ is a free parameter chosen to determine the observer's convergence rate.

By calculation and analysis, we learn that the kernel function shall satisfy the following PDE system

$$
\begin{aligned}
& p_{t}(r, s, t)=\frac{D_{s}^{-}(T(t))}{\left(R_{s}^{-}\right)^{2}}\left(p_{r r}(r, s, t)-p_{s s}(r, s, t)\right) \\
& -\lambda p(r, s, t) \\
& p(0, s, t)=0 \\
& p(r, r, t)=\frac{\left(R_{s}^{-}\right)^{2}}{2 D_{s}^{-}(T(t))} \lambda r,
\end{aligned}
$$

and the output injection gains are

$$
\begin{aligned}
& p_{1}(r, t)=-\frac{D_{s}^{-}(T(t))}{\left(R_{s}^{-}\right)^{2}}\left(p_{s}(r, 1, t)+\frac{1}{2} p(r, 1, t)\right), \\
& p_{10}(t)=\frac{3}{2}-\frac{\left(R_{s}^{-}\right)^{2}}{2 D_{s}^{-}(T(t))} \lambda .
\end{aligned}
$$

To establish the existence and regularity of the kernel function $p(r, s, t)$, we first transform the system (37) - (39) into an equivalent integral equation, and then apply the method of successive approximation.

Let $\xi=r+s, \eta=r-s$ and $q(\xi, \eta, t)=p(r, s, t)$, then we have from (37) - (39) that

$$
\begin{aligned}
& q_{t}(\xi, \eta, t)=-4 \frac{D_{s}^{-}(T(t))}{\left(R_{s}^{-}\right)^{2}} q_{\xi \eta}(\xi, \eta, t)-\lambda q(\xi, \eta, t) \\
& q(\xi,-\xi, t)=0 \\
& q(\xi, 0, t)=\frac{\left(R_{s}^{-}\right)^{2}}{4 D_{s}^{-}(T(t))} \lambda \xi .
\end{aligned}
$$


From (40),

$$
q_{\xi \eta}(\xi, \eta, t)=-\frac{\left(R_{s}^{-}\right)^{2}}{4 D_{s}^{-}(T(t))}\left(q_{t}(\xi, \eta, t)+\lambda q(\xi, \eta, t)\right) .
$$

Integrating (43) with respect to $\beta$ from 0 to $\eta$ and using boundary condition $(42)$, we have

$$
\begin{aligned}
q_{\xi}(\xi, \eta, t)= & \frac{\left(R_{s}^{-}\right)^{2}}{4 D_{s}^{-}(T(t))} \lambda-\frac{\left(R_{s}^{-}\right)^{2}}{4 D_{s}^{-}(T(t))} \\
& \times \int_{0}^{\eta}\left(q_{t}(\xi, \beta, t)+\lambda q(\xi, \beta, t)\right) d \beta .
\end{aligned}
$$

Integrating (44) with respect to $\alpha$ from $-\eta$ to $\xi$ and using (41) gives

$$
\begin{array}{r}
q(\xi, \eta, t)=\frac{\left(R_{s}^{-}\right)^{2}}{4 D_{s}^{-}(T(t))} \lambda(\xi+\eta)-\frac{\left(R_{s}^{-}\right)^{2}}{4 D_{s}^{-}(T(t))} \\
\quad \times \int_{-\eta}^{\xi} \int_{0}^{\eta}\left(q_{t}(\alpha, \beta, t)+\lambda q(\alpha, \beta, t)\right) d \beta d \alpha .
\end{array}
$$

We look for a solution of (45) in the form of

$$
q(\xi, \eta, t)=\sum_{n=0}^{\infty} q^{n}(\xi, \eta, t)
$$

where

$$
\begin{aligned}
q^{0}(\xi, \eta, t) & =\frac{\left(R_{s}^{-}\right)^{2}}{4 D_{s}^{-}(T(t))} \lambda(\xi+\eta) \\
& =\frac{\left(R_{s}^{-}\right)^{2}}{4 D_{s}^{-}\left(T\left(t_{0}\right)\right) e^{A_{D_{s}^{-}} \times \frac{1}{T\left(t_{0}\right)}}} \lambda(\xi+\eta) e^{A_{D_{s}^{-}} \times \frac{1}{T(t)}},
\end{aligned}
$$

and

$$
\begin{aligned}
& q^{n+1}(\xi, \eta, t) \\
& =-\frac{\left(R_{s}^{-}\right)^{2}}{4 D_{s}^{-}(T(t))} \int_{-\eta}^{\xi} \int_{0}^{\eta}\left(q_{t}^{n}(\alpha, \beta, t)+\lambda q^{n}(\alpha, \beta, t)\right) d \beta d \alpha .
\end{aligned}
$$

Assume that $I(t), U^{ \pm}$and thus $V(t)$ are piecewise smooth (infinitely continuously differentiable). We only consider the proof piecewisely so that both $I(t)$ and $V(t)$ are smooth in each separate time interval. Then, from (8) and the assumption that $\frac{\partial U^{ \pm}}{\partial T}$ are also smooth in the corresponding time interval, we could conclude by induction on differentiability of $T(t)$ 's derivative order that $T(t)$ is smooth as well in each time interval.

Since $T(t) \neq 0$ through all time intervals, then $\frac{1}{T(t)}$ is an analytical function in $t$. Thus, $q^{0}(\xi, \eta, t)$ is an analytical function in $t$ from the fact that the composition of analytical functions is analytical itself. Let $C$ be a positive constant such that for $t<t_{f}$,

$$
q^{0}(\xi, \eta, t) \ll C\left(1-\frac{t}{t_{f}}\right)^{-1}(\xi+\eta)
$$

with respect to $t$, uniformly for $(\xi, \eta)$, where $\ll$ denotes domination (cf. [19]). Without loss of generality we assume that $t_{f}>\frac{4}{3}$. Then

$$
q^{1}(\xi, \eta, t) \ll C C_{0}\left(1-\frac{t}{t_{f}}\right)^{-2} \frac{\xi \eta(\xi+\eta)}{2}
$$

where

$$
C_{0}=\max _{t_{0} \leq t<t_{f}}\left|\frac{R_{s}^{-2}}{4 D_{s}^{-}(T(t))}\right| .
$$

Since

$$
\int_{-\eta}^{\xi} \int_{0}^{\eta} \frac{\alpha^{n} \beta^{n}(\alpha+\beta)}{(n+1) !} d \beta d \alpha=\frac{\xi^{n+1} \eta^{n+1}(\xi+\eta)}{(n+1)(n+2) !}
$$

By induction, it could be proved that for $n \geq 1$,

$$
q^{n}(\xi, \eta, t) \ll C C_{0}^{n}\left(1-\frac{t}{t_{f}}\right)^{-n-1} \frac{\xi^{n} \eta^{n}(\xi+\eta)}{(n+1) !}
$$

Thus, the series $\sum_{n=0}^{\infty} q^{n}(\xi, \eta, t)$ could be proved to be absolutely and uniformly convergent, and the existence of $q(\xi, \eta, t)$ and $p(r, s, t)$ is established. Moreover, $p(r, s, t)$ is piecewisely analytic and has a bound

$$
\begin{aligned}
|p(r, s, t)| & =|q(\xi, \eta, t)| \\
& \leq C(\xi+\eta)\left(1-\frac{t}{t_{f}}\right)^{-1} e^{C_{0} \xi \eta\left(1-\frac{t}{t_{f}}\right)^{-1}} \\
& =2 C r\left(1-\frac{t}{t_{f}}\right)^{-1} e^{C_{0}\left(r^{2}-s^{2}\right)\left(1-\frac{t}{t_{f}}\right)^{-1}} .
\end{aligned}
$$

The transformation (32) could be verified to be invertible. Existence and regularity of its inverse could be obtained by following a similar procedure as the above proof, which is omitted here. Then, with existence, regularity of kernel function for the transformation (32) and also from invertibility of the transformation, the main theorem of this paper could be proved.

To conclude, in the equation derivation for $V(t)$, some assumptions are made:

(1). $R_{f}^{ \pm}, r_{\text {eff }}^{ \pm}$are constants,

(2). $\frac{\partial\left(U^{+}\left(\bar{c}_{s}^{+}(t)\right)-U^{-}\left(\bar{c}_{s}^{-}(t)\right)\right)}{\partial T}$ is a state-invariant, possibly timevarying, function;

and some simplification of the SPM (a polynomial solution profile) is used. In the observer design, a simplified temperature equation (17) is employed, and the following assumption is made:

(3). $I(t), U^{ \pm}$and thus $V(t)$ are piecewise smooth, and $\frac{\partial U^{ \pm}}{\partial T}$ are also smooth in the corresponding time interval.

The following theorem can then be proved.

Theorem 1: Under the above assumptions and simplifications, if choosing $\lambda<\frac{1}{4\left(R_{s}^{-}\right)^{2}} \min _{t \geq 0} D_{s}^{-}(T(t))$, then for any initial value $\tilde{w}(\cdot, 0) \in L^{2}(0,1)$, the $\tilde{w}$-system $(33)-(36)$ has an exponential stable (mild) solution $\tilde{w}(\cdot, t) \in L^{2}(0,1)$. Thus, the observer error $\tilde{c}$-system (29) - (31) is also exponentially stable in $L^{2}$ norm, which means the designed observer (25) - (28) is exponentially convergent.

\section{Simulation RESUlts}

Effectiveness of the thermal-compensated electrochemical model and performance of the proposed PDE backstepping observer for lithium-ion battery $\mathrm{SoC}$ estimation are verified by numerical simulation. The model parameters used in this simulation example are mostly cited from the publicly available lithium ion battery simulation package [20], with the 


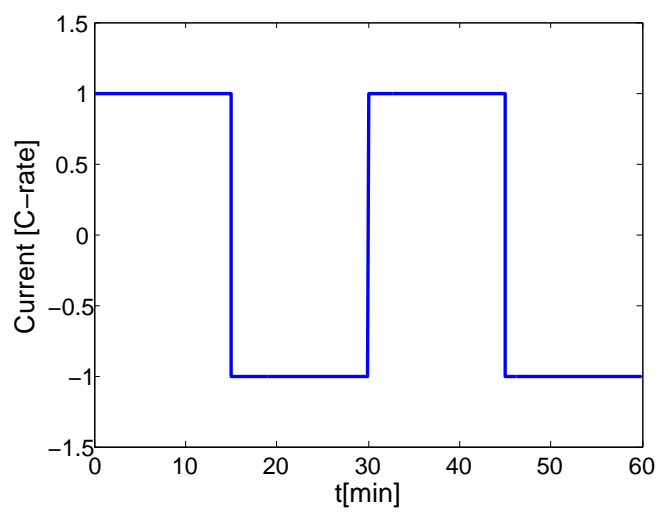

(a)

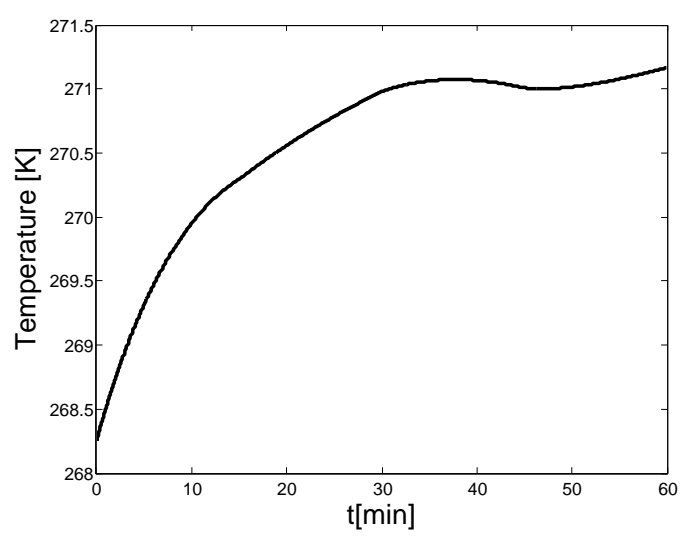

(b)

Fig. 1. Input current and output voltages for different models

rest from [12], [4], [21]. The SoC-OCV (open circuit voltage) relationship is determined by fitting our experimental data.

The input current to the battery system is a square wave alternating between 15min's constant $1 \mathrm{C}$ discharging and 15min's $1 \mathrm{C}$ charging, see, Figure 1 (a). The ambient temperature is $-5^{\circ} \mathrm{C}$. The SPM assumes constant temperature, however, the inner average temperature and battery performance have bidirectional impacts on each other. This is demonstrated from the SPM-T temperature evolution in Figure 1 (b). Since the SPM-T is more realistic, it should be able to help achieve more accurate SoC estimation.

The initial PDE observer states are set as half of the constant initial plant state values respectively, and the initial temperature for observer is set to be $-4.9^{\circ} \mathrm{C}$. A noise with covariance of $10^{-8}$ is added to the measured output voltage for both observers. Several finite difference methods are used. In Figures 2 (a) and 3 (a), the black solid lines are the true voltage and SoC values of the coupled thermalelectrochemical model; the red dashed lines are simulation results from applying the observer designed in [18] for the SPM to this coupled model; the blue dashed lines are the corresponding estimates of the observer designed in this study. Also, Figures 2 (b) and 3 (b) demonstrate the simulation results of voltage and $\mathrm{SoC}$ errors derived from

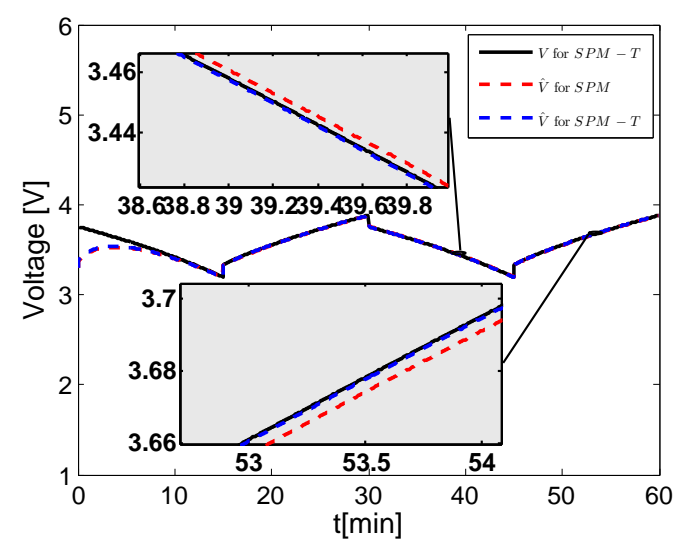

(a)

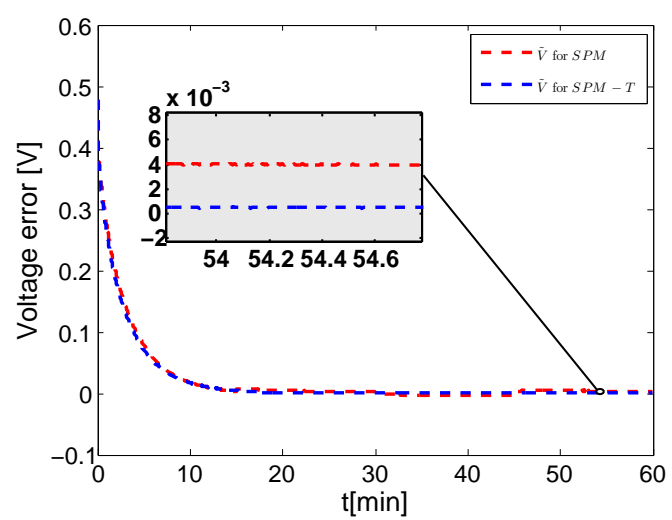

(b)

Fig. 2. Voltage true value, its estimations and estimation errors

these two different models/observers. Figures 2 and 3 show that by merging thermal behavior into the battery model and observer, we could achieve smaller voltage estimation error and more importantly, and improve the SoC estimation. This verifies the effectiveness of the method in this paper. Furthermore, Figure 4 tells that this method with the SPM-T model could achieve a good temperature estimation as well.

It is worth mentioning that for realization of the timevarying kernel function $p(r, s, t)$, we implement a composite trapezoidal rule to all integrals in the equivalent integral equation of the PDE kernel function system. By approximating the integrals with values of their integrands at equally spaced grid points, a resulting time-varying ODE for each point is derived. After we treat each point as an element of a vector and stack all points, an initial-value problem of a system of time-varying ODEs is formulated, for which we apply an implicit Euler method.

\section{CONCLUSION AND FUTURE WORK}

This paper discusses the problem of SOC estimation for the lithium-ion batteries. Compared with the SPM alone, the model we utilize in this work also incorporates a temperature equation. This model is more physics-related, and it helps to achieve a more accurate estimation of the 


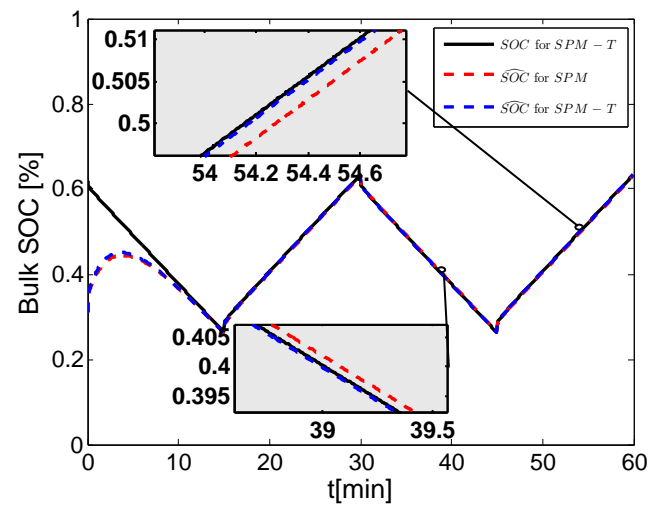

(a)

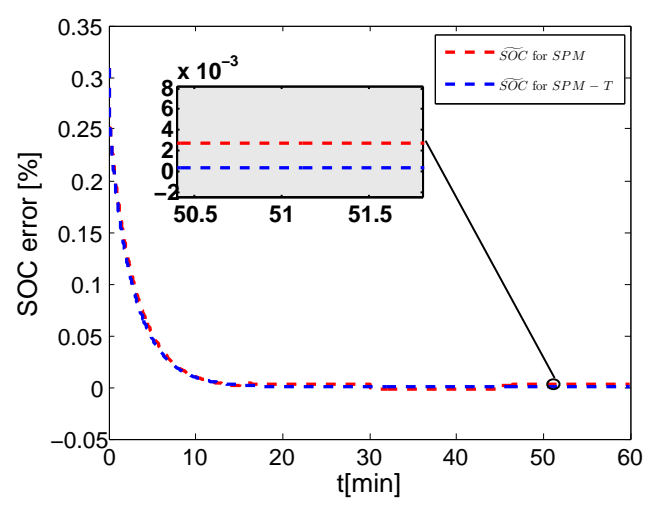

(b)

Fig. 3. SOC true value, its estimations and estimation errors

electrode lithium-ion concentration. Existence and regularity of the time-varying PDE backstepping kernel functions for the transformation between the observer error system and an exponentially stable target system are rigorously proved, then exponential stability of the observer error system is established, which proves effectiveness of the designed observer.

Some simplifications are made in this paper, and their relaxation could be directly considered as a future research direction. One possible way is to retain the cathode concentration dynamics and design its observer to work together with the available anode concentration observer from this paper. In this case, some other measurable outputs might be needed. Also, observer design for estimating the battery internal temperature itself is a valuable problem.

\section{REFERENCES}

[1] J. Chiasson and B. Vairamohan, "Estimating the state of charge of a battery," IEEE Transactions on Control Systems Technology, vol. 13, no. 3, pp. 465-470, 2005.

[2] G. L. Plett, "Extended Kalman filtering for battery management systems of LiPB-based HEV battery packs: Part 1. Background," Journal of Power sources, vol. 134, no. 2, pp. 252-261, 2004.

[3] Y. Hu and S. Yurkovich, "Battery cell state-of-charge estimation using linear parameter varying system techniques," Journal of Power Sources, vol. 198, pp. 338-350, 2012.
[4] N. Chaturvedi, R. Klein, J. Christensen, J. Ahmed, and A. Kojic, "Algorithms for advanced battery-management systems," IEEE Control Systems Magazine, vol. 30, no. 3, pp. 49-68, 2010.

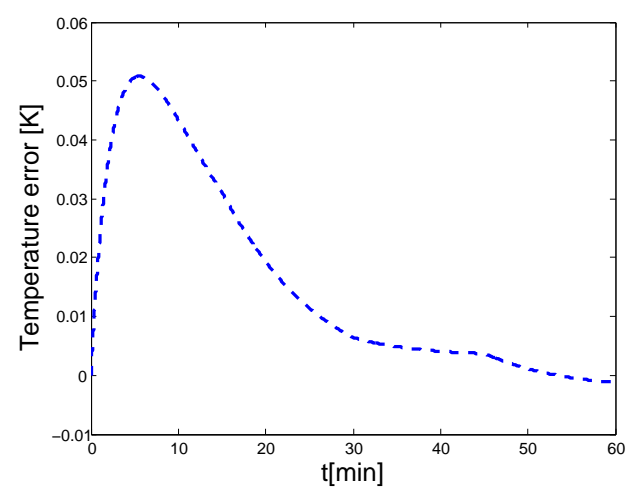

Fig. 4. Temperature estimation error

[5] R. Klein, N.Chaturvedi, J.Christensen, J. Ahmed, R. Findeisen, and A. Kojic, "Electrochemical model based observer design for a lithiumion battery," IEEE Transactions on Control Systems Technology, vol. 21, no. 2, pp. 289-301, 2013.

[6] G. G. Ning and B. N. Popov, "Cycle life modeling of lithium-ion batteries," Journal of The Electrochemical Society, vol. 151, no. 10 pp. A1584-A1591, 2004.

[7] S. Santhanagopalan, Q. Guo, P. Ramadass, and R. E. White, "Review of models for predicting the cycling performance of lithium ion batteries," Journal of Power Sources, vol. 156, no. 2, pp. 620-628, 2006.

[8] S. Santhanagopalan and R. E. White, "Online estimation of the state of charge of a lithium ion cell," Journal of Power Sources, vol. 161, no. 2, pp. 1346-1355, 2006.

[9] D. Bernardi, E. Pawlikowski, and J. Newman, "A general energy balance for battery systems," Journal of the Electrochemical Society, vol. 132 , no. 1, pp. 5-12, 1985.

[10] K. Thomas, J. Newman, and R. Darling, "Advances in lithium-ion batteries," New York, NY USA: Kluwer Academic/Plenum Publishers, ch. 12: Mathermatical Modeling of Lithium Batteries, pp. 345 - 392, 2002.

[11] C. R. Pals and J. Newman, "Thermal modeling of the lithium/polymer battery I. Discharge behavior of a single cell," Journal of the Electrochemical Society, vol. 142, no. 10, pp. 3274-3281, 1995.

[12] M. Guo, G. Sikha, and R. E. White, "Single-particle model for a lithium-ion cell: thermal behavior," Journal of the Electrochemical Society, vol. 158, no. 2, pp. A122-A132, 2011.

[13] X. Liu, Z. Chen, C. Zhang, and J. Wu, "A novel temperaturecompensated model for power li-ion batteries with dual-particle-filter state of charge estimation," Applied Energy, vol. 123, pp. 263-272, 2014.

[14] M. Krstic and A. Smyshlyaev, Boundary Control of PDEs: A Course on Backstepping Designs. SIAM, 2008.

[15] S. Tang and C. Xie, "State and output feedback boundary control for a coupled PDE-ODE system," Systems \& Control Letters, vol. 60, pp. 540-545, 2011

[16] _ - "Stabilization for a coupled PDE-ODE control system," Journal of the Franklin Institute, vol. 348, no. 8, pp. 2142-2155, 2011.

[17] V. R. Subramanian, V. D. Diwakar, and D. Tapriyal, "Efficient macromicro scale coupled modeling of batteries," Journal of the Electrochemical Society, vol. 152, no. 10, pp. A2002-A2008, 2005.

[18] S. Moura, N. Chaturvedi, and M. Krstic, "Adaptive PDE observer for battery SOC/SOH estimation via an electrochemical model," ASME Journal of Dynamic Systems, Measurement, and Control, to appear.

[19] S. Bergman and M. Schiffer, Kernel Functions and Elliptic Differential Equations in Mathematical Physics. Academic Press, 1953.

[20] J. S. Newman. Fortran programs for simulation of electrochemical systems, 2014, dualfoil.f program for lithium battery simulation. [Online]. Available: http://www.cchem.berkeley.edu/jsngrp/fortran html

[21] Wikipedia. [Online]. Available: http://www.wikipedia.org/ 